[3] Comptes rendus de l'A cadémie des Sciences, 1842.

[4] Landwirtschaftiche Versuchsstationen, 1876.

[5] Mémoires sur le lait, Paris, 1880-1884.

[6] Comptes rendus de l'Académie des Sciences, 1838.

[7] Mikroskopiske og kemiske Undersögelser over Smördamnelsen ved Kjaerningen, Copenhague, 1883.

[8] M. Beau. Sur la constitution physique des globules gras du lait. Revue générale du Lait, 1903.

[9] Milchwirtschaftliche Forschungen, 1927.

[10] Rahn et Sharp. Physik der Milehwirtschaft, Berlin, 1928.

[11] Milchwirtschaftliche Forschungen, 1929, 1930 et 1931.

[12] Munin. Neues über den Membranschleim der Fettkügelchen. Milchwirtschaftliche Zeilung, Wien, 1941.

[13] Scharz. Die Hüllen der Fettkügelchen. Molkereizeitung Hildesheim, avril 1947.

[14] Cité d'après l'article du Professeur P. Solberg Sur la structure de la crème et du beurre, paru dans Svenska Mejeritidningen des 11 et 18 octobre 1947 .

[15] Stedel. Molkereizeitung Hildesheim, 1902 et Juhresbericht der Milchwirtschattlichen Zentralstelle zu Güstrow, 1902.

[16] RАнх. Forschungen auf dem Gebiete der Milehwirtschaft, 1921.

[17] King. Westdeutsche Molkerei und Fettwirtschaft, 1944.

[18] Fritz. Beitrag zur Klärung des physikalischen Bildes der Butter. Deutsche Molkerei und Fettwirtschaft, 1944.

[19] Krvg. Globular and free fat in butter. Nederlandsche Melk en Zuiveltijdschrift, janvier-février 1947.

[20] Die Butterbildung und Struktur der Butter. Molkereizeitung Hildesheim, juillet 1947.

[21] Butterungsprocesse, neue Erkenntnisse und Anschauungen. Die Milchwissenschaft, novembre 1946.

[22] King et Fritz. Das physikalisehe Bild der Butter. Die Milchwissenscheft, janvier 1948.

\title{
ÉTAT PHYSICO-CHIMIQUE \\ DE LA XANTHINE-DÉHYDRASE DANS LE LAIT DE VACHE
}

par

M. POLONOVSKI, L. BAUDU, E. NEUZIL

(Fin.)

\section{Révélation par agitation}

Le mode d'ágitation (agitateur à pales ou secoueuse) et la nature du matériel étudié (lait total ou émulsion de crème) font apparaître quelques variantes dans la révélation de l'enzyme. 


\section{Cas du lait total.}

a) A la secoueuse (amplitude $30 \mathrm{~cm}$. - 50 mouvements par minute - température $\left.25^{\circ}\right)$.

L'activité croit régulièrement avec la durée d'agitation mais sans atteindre les chiffres obtenus par le mode de révélation par refroidissement.

b) A l'agitateur. On note d'abord une chute brusque de l'activité initiale suivie d'une remontée très lente.

Dans ce cas, le terme final de la révélation est fonction de la surface des pales de l'agitateur. La baisse d'activité du début est due à la dénaturation par frottement de la caséine dont une partie précipite au fond du récipient après avoir adsorbé une fraction de l'enzyme.

Si l'on agite ce précipité avec de l'eau distillée, celle-ci présente une certaine activité. Il s'agit bien là d'un phénomène parasite, car si l'on dispose l'agitateur de manière à faire mousser le lait, la baisse initiale d'activité est, bien plus nette : il y a ici une double adsorption : par les mousses et par la easéine précipitée.

\section{Cas des émulsions de crème.}

Les expériences donnent des résultats sensiblement identiques quel que soit le procédé d'agitation.

On note une baisse croissante de l'activité. L'agitation loin d'augmenter la stabilité de l'émulsion la détruit; de telle sorte qu'après quinze à vingt minutes cette dernière s'est fractionnée en trois phases:

a) Un très petit dépôt (formé de graisses et de protéines) qui, émulsionné dans de l'eau physiologique, présente une faible activité.

b) Une phase grasse constituée par de la crème formant de gros flocons massifs à lạ surface de la phase liquide et incapables de redonner une émulsion. L'activité de $2,8 \mathrm{~cm}^{3}$ d'une suspension à $10 \%$ de ces graisses émulsionnées dans de l'eau physiologique est de quarante minutes.

c) Une phase liquide louche. Vue au microscope, celle-ci présente de nombreux petits globules gras de 2 à $3 \mu$ formant une émulsion qui persiste plusieurs jours. L'activité de ce liquide est faible : trente minutes.

En résumé, ce procédé ne conduit pas à une révélation, car si l'on débarasse (par centrifugation à 12.000 tours), la phase liquide des globules gras qu'elle émulsionne on obtient un liquide inactif. $\mathrm{Au}$ point de vue chimique, ce liquide n'est pas acide. 


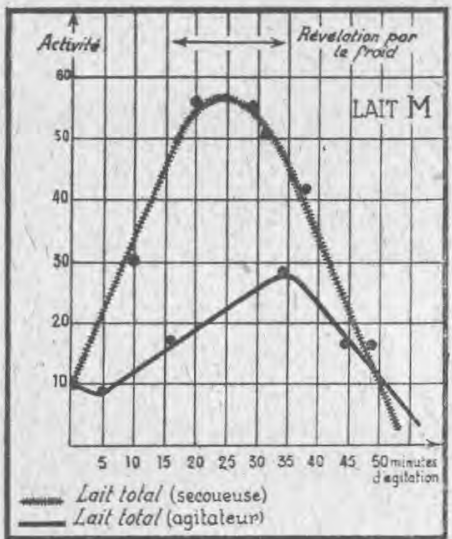

Fig. VIII.

Révélation par agitation.

Fait intéressant, nous avons conservé plusieurs semaines cette phase crémeuse sans rancissement. Phénomène que nous interprétons par la disparition des lipases et aussi par la structure de la nouvelle crème obtenue. En effet, HUNZIKER et DoRothY A., BECKEṬT avaient déjà montré que la crème contenant une forte proportion de gros globules produisait un beurre plus doux.

Cette stabilité de la crème est à rapprocher de celle que nous avions notée après déshuilage.

\section{Révélation par vieillissement}

Carrieu, Wieland et Macrae, Twigg, Worden avaient déjà remarqué l'accroissement spontané d'activité que présente un lait abandonné plusieurs heures à la température du laboratoire.

Nos expériences ont eu pour objet l'étude de laits et d'émulsions de crème.

Nous avons éliminé le rôle de l'oxygène (WORDEN) et celui de la pullulation microbienne dans la détermination du phénomène. Ce dernier se produit aussi bien dans le vide sulfurique et en présence d'antiseptiques.

Lorsque l'on utilise le thymol, l'activité croît régulièrement avec le temps pour atteindre un maximum vers la quarante-deuxième heure. A celui-ci correspond une activité voisine, bien qu'inférieure, aux chiffres de la révélation par refroidissement.

Si, avant l'expérience, on ajoute au lait $2 \%$ d'une préparation de lipase de Ricin obtenue par le procédé de WrLlstatter, la révélation est bien plus rapide.

A la lumière de ces observations, la révélation par vieillissement apparaît comme le fruit d'un processus d'hydrolyse des graisses du lait, surtout par action lipasique. L'hydrolyse bactérienne peut aussi renforcer cette action dans un lait non aseptisé, mais l'étude en devient difficile du fait de l'apparition concomitante de réductase.

Cette hydrolyse lipasique se traduit par la libération d'acides gras dans la phase liquide et la diminution de sa tension superficielle.

L'impossibilité de trouver un inhibiteur spécifique des lipases du lait qui ne diminue pas ou ne supprime pas en même temps l'activité de la xanthine déhydrase, a constitué un obstacle à l'étude 
inverse du phénomène. Le seul inhibiteur spécifique, le formol, est en même temps un substrat pour le ferment de ScHARDINGER, mais un substrat qui dénature cet enzyme si le contact est trop prolongé.

C'est pendant les processus d'hydrolyse que se produit la rupture des cénapses.

Cette action lipasique est encore confirmée par l'allure des courbes de révélation tracées au cours du vieillissement d'émulsion de erème bien lavée. Pendant plusieurs heures, on ne note pas de changement sensible. Puis soudain la révélation s'accélère. En effet, les multiples lavages nous ont débarrassés d'une partie importante de la lipase. Il en est cependant resté, adsorbé aux globules. C'est vraisemblablement à la diminution du taux de celle-ci qu'il faut attribuer ce retard à la révélation.

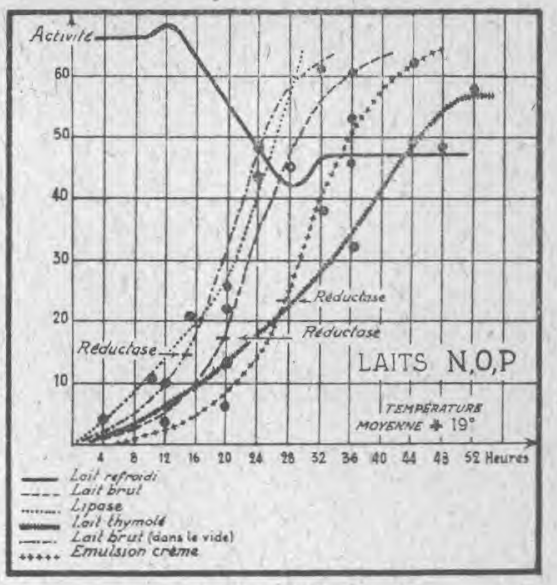

Fig. IX.

Vieillissement de laits et d'émulsions. de erème.

\section{Révélation par agents chimiques}

Avee ceux-ci, nous abordons un mode de révélation plus complexe et quelque peu mystérieux. Nous en ferons une classification artificielle : chacun d'entre eux peut, selon que l'on considère l'une ou l'autre de ses propriétés, être rattaché à un groupe ou à un autre.

Cette classification est cependant nécessaire pour l'étude :

Alealis ;

Solvants des graisses;

Corps tensio-aetifs, savons alcalins, savonsinversés, sels biliaires.

\section{A. Alcalis}

L'action révélatrice passe par plusieurs stades :

1. Début de révélation par un processus de saponification. L'alcali entre d'autant plus facilement au contact des graisses que l'élévation du $p \mathrm{H}$ du lait diminue la tendance naturelle des globules à la coalescence.

Cette action saponifiante s'exerce surtout sur les petits globules. 
Nous savons que les globules de $2 \mu$ et au-dessous résistent aux vitesses courantes de centrifugation. Si nous préparons un lactosérum en centrifugeant du lait à 6.000 tours pendant quinze minutes, nous voyons au microscope tous ces petits globules gras former une émulsion stable. L'addition de $15 \%$ de soude $M / 20$ à un tel lactosérum fait disparaître tous ces globules en quelques minutes. Il y a formation de savons.

2. Ces savons éminemment tensio-actifs viennent luxer la diastase cénapsée à la surface des globules intacts et prendre sa place (Machebcenf, Tayeau).

La solubilisation des graisses sous forme de savons est mise en évidence pan l'expérience suivante :

Lorsque, après centrifugation d'une émulsion alcalinè de crème, on neutralise par de l'acide sulfurique la phase aqueuse décantée, on voit les acides gras se libérer et se condenser en gouttelettes à la surface du liquide.

Un autre fait demeure certain, lequel établit le trait d'union entre la révélation par le froid et celle opérée par les alcalís, c'est que la saponification porte principalement sur la fraction oléique.

Lorsque l'on débarrasse par oiling off une crème de cette fraction et qu'on la lave pour chasser les dernières traces d'enzyme révélé, l'addition d'une eau alcaline ne détermine pas une nouvelle libération de diastase ou d'acides gras. On ne peut plus les mettre en ékidence dans le liquide après neutralisation à moins de prolonger le contact - crème résiduelle-alcali - plusieurs heures, ce qui permet la saponification du groupe des graisses " solides ».

Expérience réciproque, une crème traitée par les alcalis, puis décantée et Iavée, ne fournit plus d'oiling off après refroidissement.

Signalons encore la résistance très grande de l'enzyme à l'action des caustiques alcalins. Au taux où nous les avons employés, l'enzyme n'est détruit qu'après un contact de quarante à soixantes minutes.

\section{B. Solvants des graisses}

Tous ces composés agissent comme les corps tensio-actifs grâce à la structure de leur molécule. Les groupements non polaires se solubilisent dans la graisse tandis que les extrémités $\mathrm{OH}$ ou $>\mathrm{C}=\mathrm{O}$ - se tournent vers la phase aqueuse. Il s'ensuit une rupture des cénapses enzyme-graisse.

Nous avons expérimenté de multiples solvants: chloroforme, toluène, xylène, dioxane, éther, acétone, éthanol, sulfure de carbone, etc.

Les examens microscopiques d'émulsions de crème additionnées de 2 à $10 \%$ de ces solvants font apparaître après un temps variable 
un gonflement des globules gras, parfois suivi d'exsudation d'une partie de leur contenu.

L'action de ces solvants n'ést pas purement tensio-active : leur pénétration au sein du globule détermine l'apparition d'une nouvelle phase : l'équilibre du contenu globulaire ne pourra plus être représenté par un diagramme binaire. On a maintenant affaire à un système ternaire pour lequel les phénomènes de démixtion, grâce aux solubilités différentes des graisses, se produisent aux températures habituelles. Nous avons vérifié cette hypothèse par l'expérience suivante :

$10 \mathrm{~cm}^{3}$ de lait brut sont additionnés de $2 \mathrm{~cm}^{3}$ d'un solvant quelconque. On centrifuge après vingt minutes de contact et note un oiting off de moyenne importance. La phase liquide, soigneusement débarrassée de la crème est refroidie. Après quarante-cinq minutes de bain-marie, le solvant est évaporé et l'on voit apparaître, à la surface, des gouttes de graisse primitivement en solution dans le binaire eau-solvant.

\section{Corps tensio-actifs}

Il faut ici distinguer de la révélation vraie ou solubilisation de la diastase dans une phase aqueuse une fausse révélation. Cette dernière consiste en une augmentation de la finesse de l'émulsion lactée ou crémeuse par les corps tensio-actifs, sous l'influence d'une agitation même très faible.

Expérimentalement nous avons établi les points suivants : tous ces composés augmentent l'oiting off qui peut atteindre un volume supérieur à celui de la crème résiduelle.

Débarrassons une émulsion de crème de la fraction oléique par refroidissement, lavons-la et remettons-la en émulsion dans une solution à $10 \%$ de sels biliaires, par exemple. Abandonnons à la température ambiante et centrifugeons à nouveau : il se produit un nouveau déshuilage en même temps que la phase liquide est devenue active.

Ainsi il apparaît que les composés de ce groupe ont une action révélatrice plus accusée que celle du froid et atteignent des cénapses qui lui avaient échappé. C'est ainsi qu'un lait déjà révélé par le froid voit son activité encore augmentée après addition de taurocholate de sodium.

Ils favorisent par le fait même la démixtion des deux blocs gras.

Nous avons dosé les graisses extratibles de la crème par différents corps tensio-actifs. Voici-les chiffres trouvés pour différents détergents :

a) Emulsion aqueuse de $10 \mathrm{~g}$. de crème dans l'eau distillée portée 
3 heures à $-3^{\circ}$ puis centrifugée : graisses extraites par $60 \mathrm{~cm}^{3}$ d'éther (contact 8 heures) $=3,07 \mathrm{~g}$.

b) La même crème non refroidie émulsionnée dans des solutions à $10 \%$ de différents corps tensio-actifs (contact 30 minutes, puis centrifugation) :

\section{Grammes}

Sels biliaires

Taurocholate de soude ...................

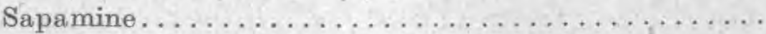

Oléate de soude $\ldots \ldots \ldots \ldots \ldots \ldots \ldots \ldots \ldots \ldots \ldots$

Sulforicinate de soude $\ldots \ldots \ldots \ldots \ldots \ldots \ldots \ldots$

Thymoxyacétate de soude.

3,22

Sur cette propriété des corps tensio-actifs de rompre les cénapses, nous avons fondé un procédé rapide d'extraction de l'enzyme. Deux faits ont attiré notre attention :

L'existence pour la plupart d'entre eux d'une dose optimum correspondant à la saturation des globules.

La dénaturation de l'enzyme par tous ces composés après un temps de contact plus ou moins long.

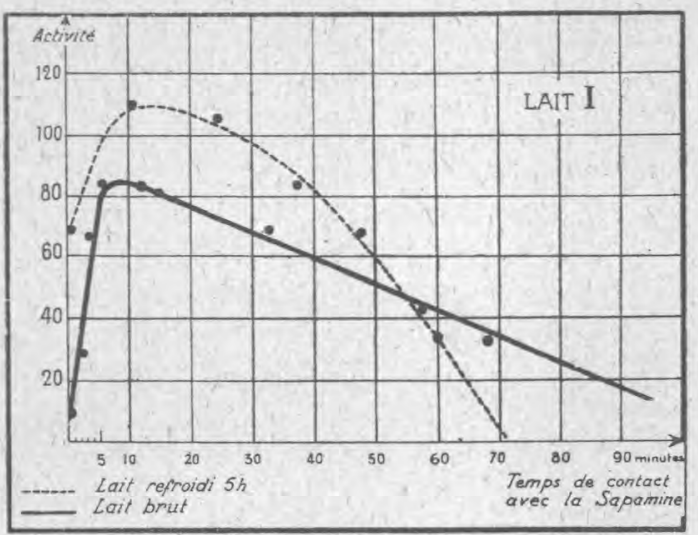

Fig. $\mathrm{X}$.

A titre d'exemple, nous donnons la courbe d'activité enzymatique d'un lait en fonetion du temps de contact avec la sapamine. (Lait, $8 \mathrm{~cm}^{3}$; sapamine à $10 \%, 4$ $\mathrm{cm}^{3}$ ) (fig. IX).

Il semble que le pouvoir dénaturant soit plus grand vis-àvis des laits refroidis que vis-à-vis des laits

Activité et temps de contact lait-sapamine. bruts.

\section{Appendice}

Nous avons dit que l'enzyme cénapsé à la surface des globules gras était actif. Or la révélation n'augmente pas la quantité totale d'enzyme d'un lait ou d'une émulsion de crème. Pourquoi dès lors l'activité augmente-t-elle?

Ceci est dû à ce que dans un milieu non révélé, la réaction se passe à la limite des couches de passage graisse-eau, e'est-à-dire à la 
surface du film protecteur de l'émulsion grasse. L'enzyme de SCHARDinger ne constitue pas à lui seul cette pellicule protectrice. Celle-ci est évidemment hérissée d'un grand nombre de groupements hydrophiles qui, si l'on considère le volume des molécules protéiques, peuvent atteindre des dimensions considérables. Cette disposition entraîne un empêchement stérique et une diminution de la vitesse de la réaction.

D'autre part, il est vraisemblable que l'enzyme est disposé en plusieurs assises moléculaires autour du globule gras, séparées les unes des autres par des feuillets de la phase dispersante.

A ce revêtement protéique extérieur au globule, appartiendrait la fraction la plus labile de l'enzyme cénapsé.

En effet, par simple lavage à l'eau distillée à $38^{\circ}$ d'une crème brute bien centrifugée, on arrive à extraire une petite fraction de l'enzyme. En remplaçant par de l'eáu distillée le lactosérum d'un lait brut d'activité quatorze minutes, on obtient ainsi après centrifugation un liquide d'activité quarante-six minutes.

\section{Conclusion}

1. L'enzyme est lié à la créme par des cénapses dont la labilité est surtout fonction de la nature des graisses.

Il est probable que la couche protéique de protection n'est pas monomoléculaire : ce qui expliquerait l'existence d'une fraction enzymatique décollable par simple lavage à l'eau distillée.

2. La révélation est la conséquence de la rupture de ces cénapses :

soit au cours de la soission par refroidissement des deux fractions isodimorphes des graisses du lait ;

soit sous l'action de corps tensio-actifs qui luxent la diastase de ses cénapses en venant prendre la place des protides.

3. Il existe une fraction d'enzyme dont les cénapses résistant à tous les moyens de scission employés. A cette fraction appartient la diastase liée aux graisses du groupe "solide».

\section{BIBLIOGRAPHIE}

\section{A. Xanthine Dehydrase}

(limitée à l'objet de cet article)

J. HorbACZew skT. Monatsch, 1891, 12, 221.

W. Spitzer. Arch. Ges. Physiol. (Pflügers), 1899, 76, 192.

F. Schardinger. Z. Untersuch. Nahr. Genussm., 1902, 5, 22.

F. Sehardinger. Chem. Ztg., 1908, 28, 1113.

R. Burian. $Z$. Physiol. Chem., 1904, 43, 497-532.

A. SohittenhelM. Z. Physiol. Chem., 1904, 42, 251. 
A. Sohitrenhelm. $Z$. Physial. Chem., 1905, 45, 121.

J. Meyer. Arb. Kais. Gesundh, 1910, 34, 115-121.

W. D. Kooper. Z, Nahr. Genussm., 1911, 20, 564.

P. H. Romer et Th. Sames. Z. Nahr. Genussm., 1911, 20, 1-10.

P. H. RoMer et Th, SAMES. Chem. Zentr., 1910, 11, 689.

W. Rulumañ, Biochem. Z., 1911, 32, 446

R. Reinhardt et E. Seibold. Biochem. Z., 1911, 31, 385-396.

J. E. Abelous et. J. Aloy. Comptés rend., 1918, 166, 130.132.

J. Aloy et Vatdigute. C. R. Soc. Biol., 1922, 85, 333.

Morgan, Stewart et Hopkins. Proe. Roy. Soe, 1922, B 94, 190.

C. VEROEst. Chem. Zentr., 1924, 11, 347.

M. Dixon et Thurlow Sylvia. Biachem. $J$., 192418 971-975.

M. Dixon et Thurlow Sylvia. Biochem. $J$. 1924, 18, 976\%

M. Dixon et Thurlow Sxlvia. Bioehem. J., 1925, $19,672$.

S. THURLOW. Biochem. J., 1925 19, 175.

R. WILDT. Milohwirtschaftliche Forshungen 1925 t. II no 5, 249-259.

M. Dixon. Biochem. J., 1925, 19, 507.

M. Dixon. Biochem. J., 1926, 20, 703.

A. Szent-GyoRGxi, Biochem. Zeitschr., 1926, 173, 275,

A. BACH et K. NikolajefF. Bio hem. Zeits hr., 1926, 169, 105.

G. VIALE. Studi Sassaresi, 1926, 3, 169-176.

B. Sbarsky et D. Mischitn. Biochem. Zeitschr., 1926, 174, 116-119.

E. J. Morgan. Biochem. J., 1926, 20, 1282.

M. Dixon et Keizo Kodama. Biochem. J., 1926, 20, 651.

H. I. Соомвs, Biochem. J., 1927, 21, 1259.

C. LIND. Le Lait, 1927, 7, n० 70, 935 .

M. Dixon. Biochem. J., 1927, 21, 1211.

T. Chrzasoz et C. Goralowna. Biochem. Z., 1927, 180, 247-262.

H. WIELANd et B. Rosenfeld. Liebig's Annalen der Chemie, 1929, CDLXXVII, $n^{\circ} 1,32$.

D. C. Haraison. Biochem. J., 1929, 23, 982.

L. Pincussen et TAkeo OYA. Biochem. Zeit., 1929, 215, 398-401.

H. Wieland et MAOrae. Liebig's Ann., 1930, 483, 217-250.

D. Mischir. Bioch. Zeitschrif, 1926, 168, 36-47.

V. Martini. Bull. Soc. Ital. Biol. spér., 1931, 6, 773-775.

A. Bovtaric et T. Jacquinot. Bull. Acad. Méd., 1933, 109, 522-525.

J. I. Tоуам. Biochem. J., 1934, 28, 1550.

D. E. Green. Biochem. J., 1934, 28, 1550.

Andersson Bengt. Arkiv. Kemi. mineral Geol., 1934, 11 A, $\mathrm{n}^{\circ} \mathrm{H} 7$.

E. J. Bigwood et J. Thomas. C. R. Soe. Biol., 1935, 118, 1639-1642.

E. J. Bigw ood et J. Thomas. C. R. Soc. Biol., 1935, 119, 337-339.

D. Keilin et E. F. Hartret. Proc. Roy. Soc., 1935-1936, B 119, 114.

E. J. Bigwood, J. Thomas et D. Wolfers. C. R. Soc. Biol., 1935, 118, 1488-1490.

R. S. TwIGG. J. of Soc, of Chem. Ind, 1937, 56, 129.

V. Н. Воотн. Biochem. J., 1938, 32, 494.

o. Warburg et W. Christian. Biochem. Z., 1938, 298, 150.

M. В. Blanch et F. С. Косн. Proc. Soc. Exptl. Biol. Méd., 1938, 38, 638. 
F. Bernheim et M. L. C. J. Bial. Chem., 1938, 123, 387.

H. S. Corran et D. E. Green. Biochem. J., 1938, 32, 2231-2240.

K. G. Weckel. Milk Dealer, 1939, 28, 94-97.

H. S. Corran, J. G. Dewan, A. H. Gordon et D. E. Green. Biochem. J., $1939,33,1694$.

E. G. Ball. J. Biol. Chem., 1939,128 51.

E. G. Ball et P. A. Ramsdell. J. Biol. Chem., 1939, 131 767-768.

I. W. Sizer et B. S. Gould. Enzymologia, 1940, 8, 75-79.

Gordon, Green, Subrahmangan. Biochem. J., 1940, 34, 764.

A. N. WORDEN. Nature, 152, 505.

TARAsSUK et Richardson. Scienee, 1941, 93, 310.

L. S. Moyer. J. Biol. Chem., 1940, 29, 133.

E. E. SNell et F. M. Strong. Enzymologia, 1939, 6, 186.

H. D. KAY. Nature, 1946, 157, 511.

M. et J. Polonovski, E. NeuziL, L. Baudu. C. R. Soc. Biol., 1947, 141, 460.

M. Polonoviki, L. Baudu, E. Neuzil. Bull. Soc. Chim. Biol., 1947, 29, 968-976.

M. Polonovisi, E. Neuzil, L. Baudu. Bull. Soc. Ohim. Biol., 1947, $29,958-957$.

\section{B. Lipase}

A. L. FLoнr. Arch. Neerland. Physiol., 1919, 3, 182-189.

E. Schlossmand. $Z$. Kinderheilk, 1922, 33, 218-222.

L. S. Patmer. J. Dairy Sc., 1922, 5, 51-63.

A. Lucca. Rivista Clinica Pediatrica, nov. 1931, 1056.

F. E. Rion et A. L. Marktey. J. Dairy Sci., 1922, 5, 64-82.

P. Rona et H. KLeInMand. Biochem. Z., 1926, 174, 18.42.

L. P. Bosman. Trans. Roy. Soc. S. Africa., 1927, 14, $255-259$.

G. Koestrer. Landw. Jahrb. Schweiz., 1928, 42, 937-966.

J. H. NATr. Ind. Eng. Chem., 1930, 22, 42-45.

R. Reder. Proc. Oklahoma Acad. Sci, 1935, 15, 49.

J. L. Hileman et E. Courtney. J. Dairy Sei., 1935, 18, 247-257.

V. N. Krukovsky et P. F. Sharp. J. Dairy Sci., 1938, 21, 671-682.

B. L. Herrington et W. Krukovsky. J. Dairy Sci., 1939, 22, 149-152.

B. L. Herrington et W. Krukovsky. J. Dairy Sci., 1939, 22, 137-147.

B. L. Herrington et W. Krukovsky. J. Dairy Sei., 1939, 22, 127-135.

W. Catel. Klin. Wochschr., 1939, 18, 342-346.

Toxoki ONo. J. Agr. Chem. Soc. (Japon), 1939, 25, $1085-1096$.

Tоуокा ONo. J. Agr. Chem. Soc. (Japon), 1939, 15, 1161-1172.

Toуoкт ONo. J. Agr. Chem. Soc. (Japon), 1940, 16, 147-158.

Richmond's. Dairy Chemistry, $4^{\mathrm{e}}$ éd. Londers, p. 175.

Menzzon. Méd. Exptl. (Ukraine), 1940, 2, 16.

D. C. Roahen et-H. H. Sommer. J. Dairy Sci., 1940, 23, 1119-1122.

V. N. KRUkovsky et P. F. SHARP. J.Dairy Sci., 1940, 23, 1119.1122.

I. A. Gould. I. Nhiry Sci, 1941, 24, 779 .

N. P. Tarassek et G. A. Richardson. Science, 1941, 93, 310-311.

M. Petwerson, M. J. Johnson et W. V. Price. Journ. Dairy Sci., 1943, 26, $233-240$. 
P. L. Kelly. J. Dairy Sci., 1945, 28, 803-820.

H. F. Wiese et L. S. Patmer. J. Dairy Sei., 1934, 17, 29-32.

G. Restivo. Pediatria, 1935, 43, 544-561.

J. A. Apptie. J. Am. Vet. Med. Assoc., 1935, 40, 331-332.

L. S. Palmer et N. P. Tarassuk. J. Dairy Sci., 1936, 19, 323-335.

A. E. SANDELIN. Chem. Zeutr., 1939, 2, 2181.

W. C. Cole et F. R. Smith. J. Dairy Sci., 1939, 22, 420-421.

N. P. Tarassuk et L. S. Palmer. J. Dairy Sci., 1939, 22, 543-558.

J. I. KeIth. Milk Dealer, 1939, 28, 82-84.

L. S. Moyer. J. Biol. Chem., 1940, 133, 29-38.

L. S. PALMer et N. P. TARAssuk. J. Dairy Sci., 1940, 23, 861-871.

V. N. KRUKovsky et P. F. SHARP. J. Dairy Sci., 1940, 23, 1109-1118.

G. Konstler, Landw. Jahrb. Sahweiz., 1940, 54, 186-194.

P. F. SHARP. J. Dairy Sci, 1940, 23, 771-782.

H. Muluer. Verslag. Landb. Onderzoek, 1945, 51, 2.

A. VAN KREVELD, Chem. Zentr., 1942, 1, 2207.

A. Van Krevet. Rec. Trav. Chim., 1946, 65, 321-328.

\section{Crémage}

H. A. Harding, F. W. Kelly et E. S. Chrisler. J. Dairy Sci, 1922, $5,468.478$.

W. H. Martin et W. B. Combs. J. Dairy Sci., 1924, 7, 197-204.

CARRIEN et CASTAGne. Le Lait, 1924, 4, no $33,188$.

A. H. SrRks. Le Lait, mai $1930,559$.

H. C. Trelogan et W. B. Combs. J. Dairy Sci., 1934, 17, 675-684.

H. ROEDER. Molkerei Ztg., 1937, 51, 634-637.

D. Le globule de graisse

K. Hatgori. J. Pharm. Soc. (Japon), 1925, 516 123-170.

O. RAHN. Milehwirtsehatfliche Forschungen 1925, 2, 383-404.

G. Achard et H. Stassano. C. R. Soe. Biol., 1925, 93, 708-710.

Ch. Porcher, Bull. Soc. Chim. Biol., 1926, 8, 997-1070.

H. H. WeIgMann. Milchwirts. Forsch., 1927, 4, 259-311.

D. A. Becketт. Ec. Proc, Roy. Dublin Soc., 1927, 2, 303-317.

Leroy Forman et I. H. SHAw. Milk Plant, 1928, 17, 36.40.

A. SCHNECK. Milehwirts. Forsch., 1928, 7, 1-34.

I. Prieger. Biochem. Z., 1930, 217, 331-336.

N. KInG. Kolloid Z., 1930, 52, 319-322.

Ch. Porcher. Lie Lait, 1930, 10, 667-682, 794-812, 900-917, 1011-1026, $1123-1137$.

Ch. Porcher. Le Lait, 1931, 11, 1-11.

N. KING. Milehwirts. Forsch., $1932,12,500-517$.

H. F. Wiese et L. S. PALMeR. J. Dairy Sci., 1932, 15, 371-381.

L. S. Patmer et H. F. Wiese. J. Dairy Sci., 1933, 16, 41-57.

W. Fritz. Milchw. Forsch., 1934, 16, 476-484.

E. Cenapses lipidoprotidiques

M. Machebde Uf. Bull. Soc. Ch. Biol., 1926, 7, 464. 
Macheb Beuf et Sandor. Bull. Soc. Ch. Biol., 1932, 14, 1168.

G. SANDOR. Thèse Doctorat Sciences, Paris, 1934.

M. Máнев вuт, Les cénapses et leur importance biólogique.

F. TAYeau. Le Lait, 1940, 20, 129.

M. Machebeuf et F. Tayeav. C. R. Ac.Sc., 1938, 206, 860.

M. Machebceuf et F. Tayeau. C. R. Soc. Biol., 1938, 129, 1181.

F. TAYEAU. Thèse Doetorat es Sciences, Bordeaux, 1939.

M. МАснев

M. Machebcur et F. TAyead. C. R. Soc. Biol, , 1940, 133, 289.

\section{F. Etude physique du lait}

H. BAUER. Biochem. Z., 1911, 32, 362-379.

W. Randale, J. M. Sherman, P. F. Sharp. J. Dairy Sei, 1927, 10, 361.371 .

W. H. E. REID. Milk Plant, 1927, 16, 39-42.

H. E. MAGEe et D. HARVEY, Biochem. J., 1926, 20, 873-883.

G. M. Batminan et P. F. Shanp. J. Agr. Research, 1928, 36, 647-674.

W. Grimer et H. Benduski. Milchw. Forsch., 1928, 7, 76-99.

M. Noкотама. Mitt. Med. Akad. Kioto, 1930, 4, 154-158.

E. O. Whittier. Journ. Biol. Chem., 1929, 1, 78 ,

S. Anselmi. Ann Chim. Applicata, 1931, 21, 454-464.

G. ROEDER, Molkerei Zeit., 1931, 45, 105.

C. Nutı. Ann. Chim. Applicata, 1934, 24, 480-483.

L. J. BURgWALD. Milk Dealer, 1935, 24, 78-79.

W. KopACZEwSKI. Le Lait, 1936, 16, 356-359.

G. BeLLe. Le Lait, 1936, 16, 13.

F. A. CARdoso et A. WANcolle. Univ. Sao-Pauls, Inst. Hig. Biol., $1939,65,24$.

L. S. Palmei et C. L. Hankinson. J. Dairy Sci., 1941, 24, 429-443.

G. Matières grasses du lait

L. Marcas et C. Hugge. Rev. Gén. du Lait, 1911, 8, 322.

R. W. Thatcher et A. C. Dahlberg. J. Agr. Research, 1917, 2, 437-450.

H. Behrendt. Biochem. Zeit., 1922, 128, 450-464.

F. Frog et Schmidt, S. Nielsen. Biochem. Zeit., 1922, 127, 163-173.

O. RAHN. Milch. Forsch., 1925, 2, 383-404.

H. Corblin. Bull. Soc. en cour. ind. nat., 1928, 232-242.

H. A. Ruehe et B. A. Strritz. J. Dairy Sei., 1925, 8, 330-343.

E. Weaver et C. A. Matthews. Ia. Agr. Expt. Sta. Research. Bull, $1928,107,158-180$.

W. VAN DAM. Le Lait, $1928,8,369$.

I. S. Popow et S. P. Nrkolsky. Bull. Rech. Inst., 300 t, Moscou, $1928,4,24$.

F. P. Sanmann et H. A. Ruehe. J. Dairy Sdi., 1930, 13, 48-63.

A. Petrik. Molkerei $Z$ g., 1931, 45, 2259-2263.

M. TEgoni. Annali d'I giene, 1928, 403.

E. O. Whittiek et A. G. Benton. J. Dairy Sci., 1927, 10, 126-138, 
W. VAN DAMr. Chem. Zentr., 1927, 2, 1106.

G. ECKARDt. Molkerei Ztg., 1933, 1591-1592.

H. E. Ross. Milk Plant, 1938, 27, 30-31.

K. G. Weckel. Can. Dairy and Iee Cream J., 1939, 18, 30-36.

A. H. Rishot et P. F. ShaRP. J. Dairy Sci., 193821 683-687.

W. MoHr et E. EYsANK. Forschungsdienst 1940, 10, 579-586.

F. TAyent. Le Lait, 1940, 21, 129-136.

P. Hindich. Paul S., Gunde B. G., Maddison L., J. Soc. Chim. Ind., $1940,59,138-144$.

G. M. Trout et M. V. Scheid. J. Dairy Sci., 1943, 26, 619-623.

\title{
PHYSICO-CHIMIE DU LAIT
}

\author{
par \\ W. KOPACZEWSKI \\ IV. DISJONCTION DU LAIT
}

\section{A. Introduction}

Jusqu'à présent nous avons examiné la gélification et la stabilisation du lait. Nous allons aborder l'étude d'un phénómène irréversible, conduisant à la destruction totale de l'équilibre colloïdal de ce liquide biologique, que nous avons dénommé, en 1943, disjonction.

Les globules des matières grasses du lait, dont le diamètre oscille entre $10 \mu$ et $1 \mu 6$, communiquent au lait sa couleur blanche ; leur taux atteint environ $3,6 \%$; elles se composent essentiellement $(3,6 \%)$ de glycérides saturés d'acides gras (butyrique, palmitique et oléique), de 0,5 à $1,0 \%$ de matières insaponifiables (cholestérol) et de $1,0 \%$ de lécithine. Ces matières forment une émulsion au sein d'un liquide intermicellaire, chaque gouttelette étant entourée d'une eouche protectrice.

Une agitation énergique permet de diviser ces gouttelettes, de sorte que chacune d'elles peut former 1.200 particules nouvelles. Cette émulsion est très stable : une centrifugation la plus énergique n'aboutit pas à la séparation de ees particules et, eo ipso, à la décoloration complète du lait. Un travail de W. M. ORD, publié en 1879, démontre qu'une agitation d'une dispersion d'albumine dans l'huile provoque la formation d'une couche protectrice autour de chaque micelle ce qui rend cette émulsion très stable, tout comme celle du lait. C'est pourquoi le lait de femme, pauvre en protides, peut être dégraissé par l'agitation avec de l'éther ; ce n'est pas le cas du. lait de vache, beaucoup plus riche en protides.

R.AHN, en étudiant la composition du lait écrémé, d'une part, 